\title{
Dosimetric Analysis with Intensity-modulated Radiation Therapy for Central Nervous System Irradiation in Patients with Brain Cancer Compared with Three- dimensional Conformal Radiation Therapy Treatment
}

\author{
Noor S. Omar*, Ronak T. Ali \\ Department of Basic Sciences, Biophysics Unit, College of Medicine, Hawler Medical University, Erbil, Kurdistan Region, Iraq
}

\author{
${ }^{*}$ Corresponding author: \\ Noor S. Omar, \\ Master student in \\ Department of Basic \\ Sciences, Biophysics Unit, \\ College of Medicine, Hawler \\ Medical University, Erbil, \\ Kurdistan Region, Iraq. \\ E-mail: biophysics111@ \\ gmail.com
}

Received: 29 July 2019

Accepted: 14 October 2019

Published: 01 December

2019

\section{DOI}

10.25156/ptj.v9n2y2019.pp50-54

\begin{abstract}
A B S T R A C T
Intensity-modulated radiation therapy (IMRT) was put as the development of three-dimensional conformal radiation therapy (3D-CRT). The purpose of the present study is to compare the dosimetric analysis of two techniques of radiotherapy (IMRT) and 3D-CRT, which include target volume and organ at risk for both plans. The present study enrolled that nine patients with different types of brain cancer which previously irradiated from November 2018 to May 2019 were selected in Zhianawa Cancer Center in Sulaymaniyah; all cases were planned again by both techniques 3D-CRT and IMRT. IMRT planning provides reducing the dose of both right and left optic nerve mean dose for right optic nerve $13.70 \mathrm{~Gy}$ and left $14.93 \mathrm{~Gy}$ compared with the 3D-CRT plan (right optic nerve $23.54 \mathrm{~Gy}$ and left $19.13 \mathrm{~Gy}$ ). $P=0.2$ for the right optic nerve and $P=0.56$ for the left optic nerve were statistically significant. IMRT plan reduces dose to the optic chiasm compared to 3D-CRT plan, the mean dose of optic chiasm for IMRT was 33.37 Gy relative to 3D-CRT which was $34.28 \mathrm{~Gy}$ and $P=0.92$. IMRT plan was better than 3D-CRT for many organs at risk, especially for optic chiasm and both optic nerve deliver less dose than 3D-CRT.
\end{abstract}

Keywords: Brain cancer; Intensity-modulated radiation therapy; Radiotherapy; Reduce dose; Threedimensional conformal radiation therapy

\section{INTRODUCTION}

Intensity-modulated radiation therapy (IMRT) was put as the development of three-dimensional conformal radiation therapy (3D-CRT) (Bentzen, 2005; Bortfeld, 2006) to improve tumor control and patient survival (Navarria et al., 2014). The target of radiotherapy is to deliver the prescribed dose to the tumor and organs at risk (OAR), receiving a minimum dose as much as possible. Nowadays, a different type of tumors is treated by radiotherapy with surgery and chemotherapy (Chui et al., 2001).

The tumors can affect the nervous system (Giglio and Gilbert, 2010). Nearly half of the patients who have cancer will receive radiotherapy as a section of their oncologic treatment (Kumar et al., 2013). 3D-CRT uses computed tomography to produce three-dimensional volumes of a patient's anatomy (Goyal., 2008) and also to visualize OAR and the tumor (GhoshLaskar et al., 2016; Nutting et al., 2011).

3D-CRT is a very available radiotherapy technique which considered as standard (Kortmann et al., 2003; Krasin et al., 2010; Chan, 2015).
Non-uniform radiation beam intensities use in IMRT by computer-based optimization to achieve better dose distribution (Tejpal et al., 2010). Furthermore, multileaf collimators that use in IMRT are dynamic or static, which delivers high doses while minimizing dose to the surrounding healthy tissues (Bhatnagar et al., 2006; Zelefsky et al., 2002; Heron et al., 2003). Distribution of dose is inversely determined in IMRT, which means that the treatment planner must decide the dose distribution for each organ at risk and after that, the computer will start calculating a group of the beam that will be generated (Spirou and Chui, 1998) and also is expected to improve radiotherapy by late morbidity of treatment and better covers the volume of the target (Lin et al., 2003; Scott-Brown et al., 2010).

IMRT contains the radiation field, in which the shape is in accordance with the projective shape of planning target volume (PTV) in the radiation beam. And then, multiple fixed angle beams are usually needed for better qualities (Xie et al., 2014), which patient's quality of life can be more improved (Sun et al., 2014; Zhou et al., 2017; Kuang et al., 2012). 
The purpose of the present study is to compare the dosimetric analysis of two techniques of radiotherapy IMRT and 3D-CRT, which include target volume and organ at risk for both techniques.

\section{MATERIALS AND METHODS}

\section{Patient's Selection}

All of the patient's ages were ranged between 9 and 52 years, three males and six females. The prescribed dose for all the cases was 54 Gy with 30 fractions.

\section{Forward Planning (3D-CRT)}

The present study uses Xio (release 5.00.02) threedimensional radiotherapy treatment planning system, the energy used to produce a plan in 3D-CRT for all types of brain cancer was $6 \mathrm{MV}$ photon. Three to four beams were designed for treatment planning. There are three main volumes to be into account in radiotherapy planning, the first volume is the position of the tumor and this is known as the gross tumor volume (GTV). The second volume surrounds the GTV and describes the extent of microscopic unexpected tumor spread; this is known as the clinical target volume (CTV). The original concepts of the GTV and CTV were detailed in report 50 from the International Commission on Radiation Units and Protection (ICRU), in 1993 (ICRU Report 50). In the third volume, the PTV is a geometric concept designed to confirm that the radiotherapy prescription dose is actually delivered to the CTV (Burnet et al., 2004). To cover, the PTV multileaf collimator (MLC) was used. A wedge also used to perform dose homogeneity in PTV.

\section{Inverse Planning (IMRT)}

Seven-field and nine-field were used for inverse planning with the energy of 6MV photon. For seven fields were used to cover PTV using (MLC) at gantry angle $\left(80^{\circ}, 120^{\circ}, 160^{\circ}\right.$, $200^{\circ}, 240^{\circ}, 280^{\circ}$, and $\left.300^{\circ}\right)$. For nine fields were used to cover PTV using (MLC) at gantry angle $\left(0^{\circ}, 40^{\circ}, 80^{\circ}, 120^{\circ}\right.$, $160^{\circ}, 200^{\circ}, 240^{\circ}, 280^{\circ}$, and $320^{\circ}$ ).

\section{The Tolerance Doses for OAR for Both Parallel and Serial Organs}

The maximum dose for OAR for the lens was $7 \mathrm{~Gy}$, the optical nerve was $55 \mathrm{~Gy}$, mean dose for cochlea was $\leq 45 \mathrm{~Gy}$, mean dose for the eye was $<35 \mathrm{~Gy}$, brain steam was $<54 \mathrm{~Gy}$, and optic chiasm $55 \mathrm{~Gy}$.

\section{Prescription Dose and Dosimetric Analysis}

The prescription dose for all patients was 54 Gy in 30 fractions, for each day, the patient received $1.8 \mathrm{~Gy}$. Dosevolume histograms (DVHs) of both techniques used to evaluate maximum dose, mean dose for each organ at risk, and cover of PTV. All plans in both techniques depend on DVHs to ensure that the $95 \%$ of the volume of PTV received $95 \%$ of the prescribed dose.

The DVHs are a tool to show the dose that is delivered to OAR and volume of the target (Guckenberger et al., 2006). Conformity index (CI) defines as an attempt to measure exactly how well the distribution of dose follows the shape of the target volume, and it is a ratio of the tissue volume which receives at least $95 \%$ of the prescription dose divided by the volume of the PTV, as shown in equation (1). CI is more conformal when its value closer to 1 (Foroudi et al., 2012).

$$
\mathrm{CI}=\mathrm{V}_{95 \%} / \mathrm{VPTV}
$$

Homogeneity index (HI) is a common tool that is used to analyze dose homogeneity in the tumor volume, as shown in equation (2). It is used to compare the dose distributions of many treatment plans (Feuvret et al., 2006; Gong et al., 2008; Wu et al., 2003).

$$
\mathrm{HI}=\mathrm{D}_{2 \%}-\mathrm{D}_{98 \%} / \mathrm{D}_{50 \%}
$$

Where $\mathrm{D}_{2 \%}$ and $\mathrm{D}_{98 \%}$ represent the doses of the PTV, respectively, $\mathrm{D}_{98 \%}$ means that at least $98 \%$ of the PTV receives this dose, and hence, $\mathrm{D}_{2 \%}$ means that at least $2 \%$ of the PTV receives this dose. $\mathrm{D}_{2 \%}$ is considered to be the maximum dose and $\mathrm{D}_{98 \%}$ is considered to be the minimum dose, lower HI values mean a more homogenous target dose (Yoon et al., 2007).

\section{Statistical Methods}

Statistical analysis was done using SPSS version 19 (IBM) statistical software package. Data described by the mean \pm SD. $t$-test was used to compare the prescribed dose of both technique, and $P<0.05$ was considered to be statistically significant.

\section{Ethical Considerations}

The present study was approved by the Research Ethics Committee of the College of Medicine at Hawler Medical University.

\section{RESULTS}

Table 1 represents the clinical characteristics of the patients.

IMRT planning provides reducing the dose of both right and left optic nerve mean dose for (right was 13.7 $\pm 12.15 \mathrm{~Gy}$ and left $14.93 \pm 14.19 \mathrm{~Gy}$ ) compared with 3D-CRT (right was $23.54 \pm 18.77 \mathrm{~Gy}$ and left $19.13 \pm$ 16.15 Gy), as shown in Figures 1 and 2.

3D-CRT shows reduce dose received to left cochlea, it was 15.8 Gy relative to IMRT that was 18.1 Gy. However, the 
mean dose for the right cochlea was 20.01 Gy in IMRT and 21.14 Gy in 3D-CRT, as shown in Figures 3 and 4.

IMRT plan reduces dose to the optic chiasm compared to 3D-CRT plan, the mean dose of optic chiasm was $33.37 \mathrm{~Gy}$ relative to 3D-CRT which was 34.28 Gy. However, the 3D-CRT plan shows reduce dose received to the brain stem (mean dose was 37.18 Gy relative to IMRT), which was $41.4 \mathrm{~Gy}$, as shown in Figures 5 and 6.

\section{Table 1: Clinical characteristics of the patients}

Cases location of tumor volume of PTV $\left(\mathrm{cm}^{3}\right)$ tumor type

1. The middle part of the brain 290.128 brain stem glioma

2. The lower middle part of the brain 128.708 diffuse intrinsic pontine glioma

3. The right part of the brain 405.964 anaplastic ependymoma GIII

4. The right part of the brain 331.533 supine brain tumor

5. The right back part of the brain 78.695 meningioma

6. The upper middle part of the brain 181.568 meningioma

7. The right part of the brain 345.713 oligodendroglioma GII

8. The middle back part of the brain 77.495 ependymoma GII (Cerebellar)

9. The middle part of the brain 22.690 pituitary adenoma

PTV: Planning target volume

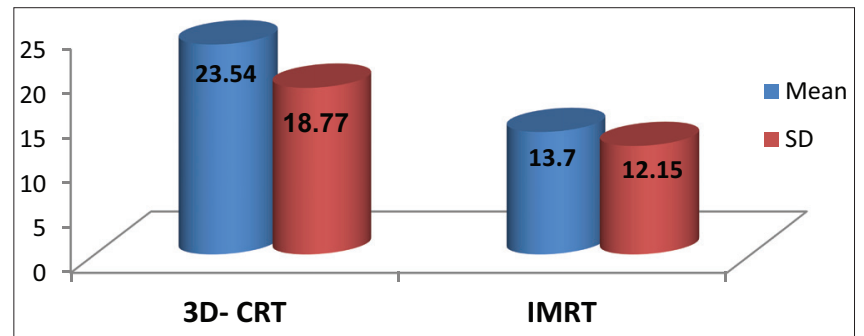

Figure 1: Mean \pm SD of the maximum dose for right optic nerve in IMRT versus 3D-CRT

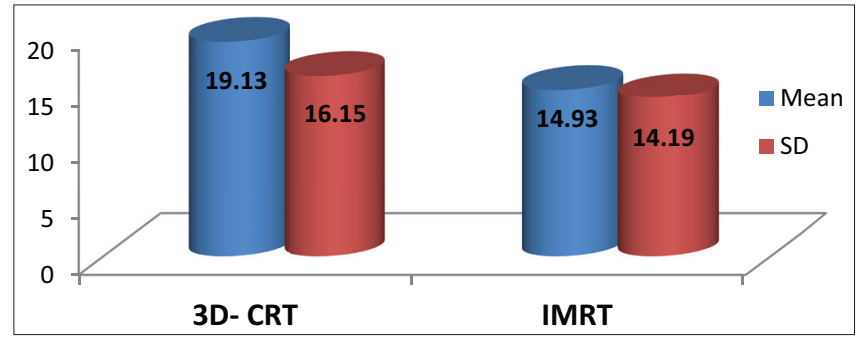

Figure 2: Mean and standard deviation $\left(\mathrm{SD}_{ \pm}\right)$maximum dose for left optic nerve in IMRT versus 3D-CRT

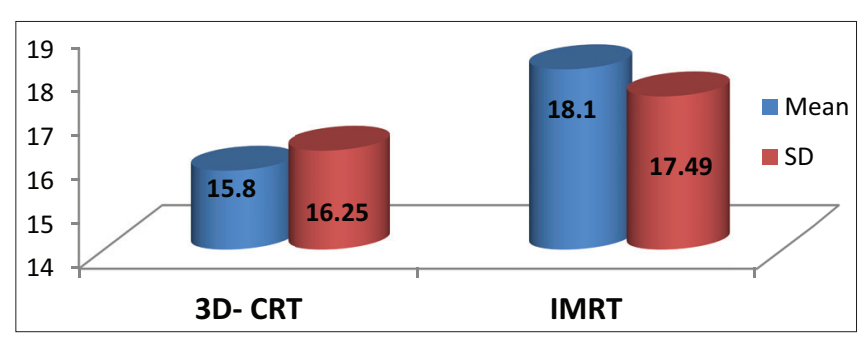

Figure 3: Mean and standard deviation (SD \pm ) maximum dose for left cochlea in IMRT versus 3D-CRT
The mean dose of the right eye for both plans was slightly different for 3D-CRT was 13.15 Gy and for IMRT was 13.27 Gy. However, for the left eye, the mean dose was 8.41 Gy and for IMRT was 12.73 Gy. For the left lens, 3D-CRT shows reduce mean dose than IMRT for both was 2.46 Gy and 3.44 Gy.

IMRT plan was better for reducing the mean dose for the right lens, it was $2.88 \mathrm{~Gy}$ compared with 3D-CRT that mean dose was $6.75 \mathrm{~Gy}$, as shown in Figure 7.

The organ at risk for both techniques was not statistically significant, right and left eye, $P=0.98$ and 0.65 while for right and left cochlea, $P=0.89$ and 0.75 . However, for both lenses, $P=0.48$ and 0.56 was statistically significant. However, for both optic nerves $P=0.2$ and 0.56 , for brain stem $P=0.7$, and optic chiasm $P=0.92$ are shown in Table 2 .

Mean and standard deviation of CI for both plan 3D-CRT and IMRT was $0.97 \pm 0.01,0.87 \pm 0.31$, and $P=0.33$, which is not statistically significant, while $\mathrm{HI}$ for 3D-CRT was $0.10 \pm 0.04$ and for IMRT was $0.12 \pm 0.03,(P=0.195) . \mathrm{D}_{5 \%}$ and $\mathrm{D}_{2 \%}$ refer that volume (PTV) received $5 \%-2 \%$ of the

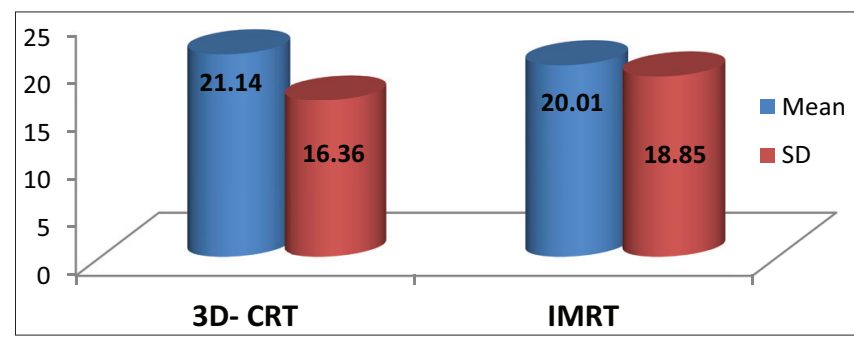

Figure 4: Mean and standard deviation ( $S D \pm$ ) maximum dose for right cochlea in IMRT versus 3D-CRT

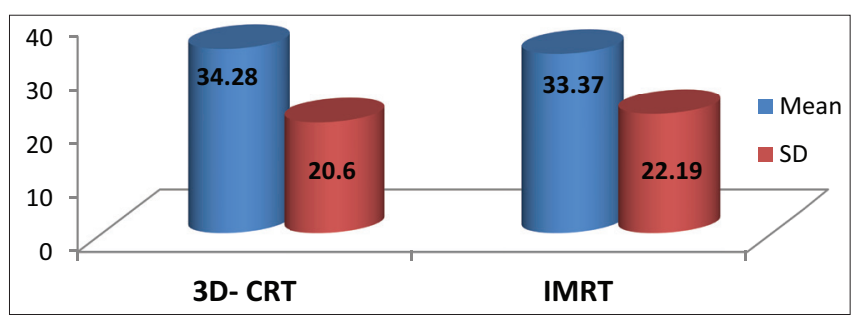

Figure 5: Mean and standard deviation ( $\left.\mathrm{SD}_{ \pm}\right)$maximum dose for optic chiasm in IMRT versus 3D-CRT

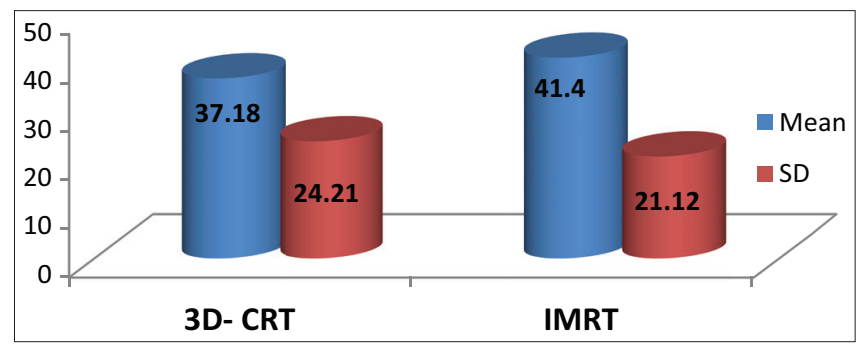

Figure 6: Mean and standard deviation $\left(\mathrm{SD}_{ \pm}\right)$maximum dose for brain stem in IMRT versus 3D-CRT 
Table 2: The mean dose of radiotherapy to organs at risk using different radiation modality

\begin{tabular}{lccc}
\hline Organ at risk & 3D-CRT Mean \pm SD & IMRT Mean $\pm S D$ & $P$-value \\
\hline Right eye & $13.15 \pm 19.31$ & $13.27 \pm 16.64$ & 0.98 \\
Right cochlea & $21.14 \pm 16.36$ & $20.01 \pm 18.85$ & 0.89 \\
Right lens & $6.75 \pm 16.08$ & $2.88 \pm 1.70$ & 0.48 \\
Right optic nerve & $23.54 \pm 18.77$ & $13.70 \pm 12.15$ & 0.2 \\
Brain stem & $37.18 \pm 24.21$ & $41.40 \pm 21.12$ & 0.7 \\
Optic chiasm & $34.28 \pm 20.60$ & $33.37 \pm 22.19$ & 0.92 \\
Left eye & $8.41 \pm 13.08$ & $12.73 \pm 15.52$ & 0.65 \\
Left cochlea & $15.80 \pm 16.25$ & $18.10 \pm 17.49$ & 0.75 \\
Left lens & $2.46 \pm 4.37$ & $3.44 \pm 2.31$ & 0.56 \\
Left optic nerve & $19.13 \pm 16.15$ & $14.93 \pm 14.19$ & 0.56 \\
\hline
\end{tabular}

3D-CRT: Three-dimensional conformal radiation therapy, IMRT: Intensitymodulated radiation therapy

Table 3: Dosimetric parameters of 3D-RT and IMRT plan

\begin{tabular}{lccc} 
Dosimetric & 3D-CRT & $\begin{array}{c}\text { IMRT } \\
\text { parameters }\end{array}$ & $P$-value \\
\hline Conformity index & $0.97 \pm 0.01$ & $0.87 \pm 0.31$ & 0.33 \\
Homogeneity index & $0.10 \pm 0.04$ & $0.12 \pm 0.03$ & 0.195 \\
$\mathrm{D}_{98 \%}$ & $50.95 \pm 1.04$ & $51.21 \pm 0.67$ & 0.3 \\
$\mathrm{D}_{95 \%}$ & $52.07 \pm 0.49$ & $52.17 \pm 0.63$ & 0.45 \\
$\mathrm{D}_{5 \%}$ & $56.97 \pm 1.09$ & $57.58 \pm 0.99$ & 0.013 \\
$\mathrm{D}_{2 \%}$ & $57.34 \pm 1.21$ & $58.06 \pm 1.12$ & 0.007 \\
\hline
\end{tabular}

3D-CRT: Three-dimensional conformal radiation therapy, IMRT: Intensitymodulated radiation therapy

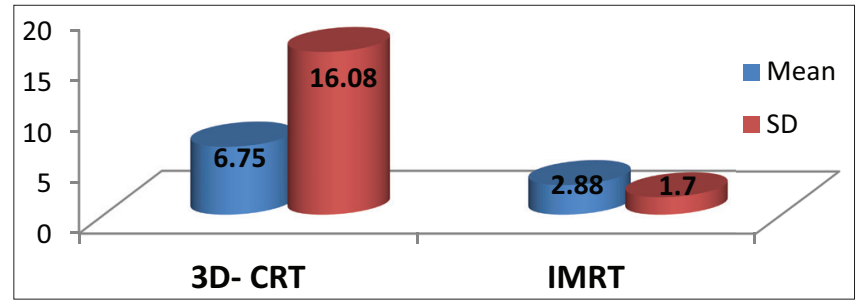

Figure 7: Mean and standard deviation $\left(\mathrm{SD}_{ \pm}\right)$maximum dose for the right lens in IMRT versus 3D-CRT

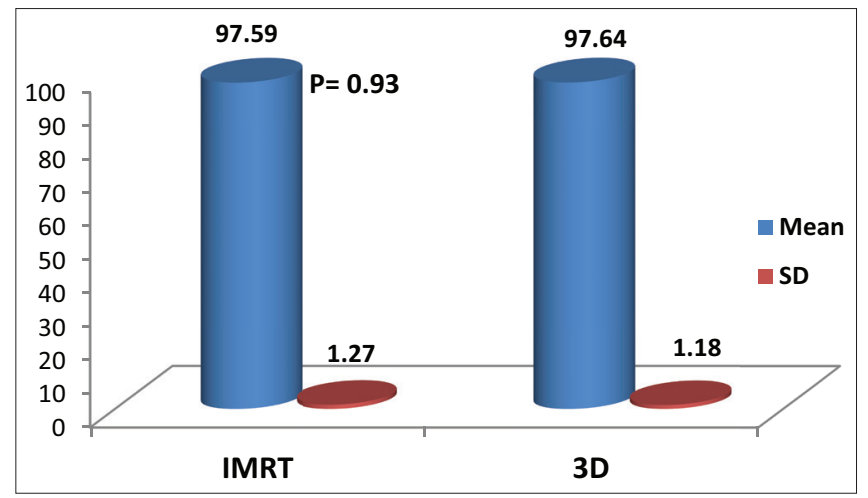

Figure 8: Mean and SD of PTV in 3D vaersus IMRT among brain tumors

prescribed dose, $\mathrm{D}_{5 \%}$ and $\mathrm{D}_{2 \%}$ were significant difference in IMRT compared with 3D-CRT and $P=0.013$ for $\mathrm{D}_{5 \%}$ and 0.007 for $\mathrm{D}_{2 \%}$ which is statistically significant, as shown in Table 3.
PTV for both plans was not statistically significant, which for IMRT was $97.59 \%$ and for 3D-CRT was $97.64 \%$ and $P=0.93$, as shown in Figure 8.

\section{DISCUSSION}

This study shows the dosimetric comparison between 3D-CRT plan and IMRT plan, resulting in the present study shows that both right and left optic nerves receive a less prescribed dose in the IMRT plan as relative to 3D-CRT, but for brain stem, 3D-CRT shows reduce dose received than IMRT. However, this result compares with a study done in Egypt by Al Zayat et al. and shows that in IMRT optic nerve receives a higher dose (38.86 Gy) than 3D-CRT (27.57 Gy). However, for brain stem, IMRT shows better pan (72.17 Gy) relative with 3D-CRT (73.05 Gy) (Al Zayat et al., 2014).

In 3DCRT plan which usually use two or three fields with wedges in relative with the IMRT plans which using more than three fields, using a small number of the fields given advantages to the 3D-CRT plan which is less monitor units, short time of treatment and make small low-dose areas. This is crucial because low-dose areas may induce secondary cancer (Moret et al., 2009; Fontenot et al., 2009; Zwahlen et al., 2009).

\section{CONCLUSION}

IMRT plan was better than 3D-CRT for many OAR, especially for optic chiasm and both optic nerves deliver less dose than 3D-CRT, while PTV for both plans was slightly different. Both techniques can use to treat brain cancer, but if OARs were so close to PTV that it is better to use IMRT than 3D-CRT.

\section{CONFLICTS OF INTEREST}

The authors reported no conflicts of interest.

\section{REFERENCES}

Al Zayat, D. M., E. M. Attalla, H. S. Abouelenein, Y. M. Elkem and W. Khalil. 2014. Dosimetric comparison of intensity-modulated radiotherapy versus three-dimensional conformal radiotherapy for patients with brain tumors. Open J. Radiol. 4(01): 85.

Bentzen, S. M. 2005. Radiation therapy: Intensity modulated, image guided, biologically optimized and evidence based. Radiother. Oncol. 77(3): 227-230.

Bhatnagar, A. K., E. Brandner, D. Sonnik, A. Wu, S. Kalnicki, M. Deutsch and D. E. Heron. 2006. Intensity modulated radiation therapy (IMRT) reduces the dose to the contralateral breast when compared to conventional tangential fields for primary breast irradiation. Breast Cancer Res. Treat. 96(1): 41-46.

Bortfeld, T. 2006. IMRT: A review and preview. Phys. Med. Biol. 51(13): R363. 
Burnet, N. G., S. J. Thomas, K. E. Burton and S. J. Jefferies. 2004. Defining the tumour and target volumes for radiotherapy. Cancer Imaging. 4: 153-161.

Chan, M. D. 2015. Recent technical advances and indications for radiation therapy in low-grade glioma. Semin. Radiat. Oncol. 25: 189-196.

Chui, C. S., M. F. Chan, E. Yorke, S. Spirou and C. C. Ling. 2001. Delivery of intensity-modulated radiation therapy with a conventional multileaf collimator: Comparison of dynamic and segmental methods. Med. Phys. 28(12): 2441-2449.

Feuvret, L., G. Noël, J. J. Mazeron and P. Bey. 2006. Conformity index: A review. Int. J. Radiat. Oncol. Biol. Phys. 64(2): 333-342.

Fontenot, J. D., A. K. Lee and W. D. Newhauser. 2009. Risk of secondary malignant neoplasms from proton therapy and intensity-modulated $\mathrm{x}$-ray therapy for early-stage prostate cancer. Int. J. Radiat. Oncol. Biol. Phys. 74(2): 616-622.

Foroudi, F., L. Wilson, M. Bressel, A. Haworth, C. Hornby, D. Pham, J. Cramb, S. Gill, K. H. Tai and T. Kron. 2012. A dosimetric comparison of $3 \mathrm{D}$ conformal vs intensity modulated vs volumetric arc radiation therapy for muscle invasive bladder cancer. Radiat. Oncol. 7(1): 111.

Ghosh-Laskar, S., P. H. Yathiraj, D. Dutta, V. Rangarajan, N. Purandare, T. Gupta, A. Budrukkar, V. Murthy, S. Kannan and J. P. Agarwal. 2016. Prospective randomized controlled trial to compare 3-dimensional conformal radiotherapy to intensitymodulated radiotherapy in head and neck squamous cell carcinoma: Long-term results. Head Neck. 38(S1): E1481-E1487.

Giglio, P. and M. R. Gilbert. 2010. Neurologic complications of cancer and its treatment. Curr. Oncol. Rep. 12(1): 50-59.

Gong, Y., J. Wang, S. Bai, X. Jiang and F. Xu. 2008. Conventionallyfractionated image-guided intensity modulated radiotherapy (IG-IMRT): A safe and effective treatment for cancer spinal metastasis. Radiat. Oncol. 3(1): 11.

Goyal, S., A. Cohler, J. Camporeale, V. Narra and N. J. Yue. 2008. Intensity-modulated radiation therapy for orbital lymphoma. Radiat. Med. 26(10): 573-581.

Guckenberger, M., F. Pohl, K. Baier, J. Meyer, D. Vordermark and M. Flentje. 2006. Adverse effect of a distended rectum in intensity-modulated radiotherapy (IMRT) treatment planning of prostate cancer. Radiother. Oncol. 79(1): 59-64.

Heron, D. E., K. Gerszten, R. N. Selvaraj, G. C. King, D. Sonnik, H. Gallion, J. Comerci, R. P. Edwards, A. Wu and R. S. Andrade. 2003. Conventional 3D conformal versus intensity-modulated radiotherapy for the adjuvant treatment of gynecologic malignancies: A comparative dosimetric study of dose volume histograms. Gynecol. Oncol. 91(1): 39-45.

ICRU. 1999. Prescribing, Recording and Reporting Photon Beam Therapy. Report 50. International Commission on Radiation Units and Measurements, Bethesda, MD.

Kortmann, R. D., B. Timmermann, R. E. Taylor, G. Scarzello, L. Plasswilm, F. Paulsen, B. Jeremic, A. K. Gnekow, K. Dieckmann and S. Kay. 2003. Current and future strategies in radiotherapy of childhood low-grade glioma of the brain. Strahlenther. Onkol. 179(8): 509-520.

Krasin, M. J., A. M. Davidoff, X. Xiong, S. Wu, C. H. Hua, F. Navid, C. Rodriguez-Galindo, B. N. Rao, K. A. Hoth and M. D. Neel. 2010. Preliminary results from a prospective study using limited margin radiotherapy in pediatric and young adult patients with high-grade nonrhabdomyosarcoma soft-tissue sarcoma. Int. J. Radiat. Oncol. Biol. Phys. 76(3): 874-878.

Kuang, W. L., Q. Zhou and L. F. Shen. 2012. Outcomes and prognostic factors of conformal radiotherapy versus intensity-modulated radiotherapy for nasopharyngeal carcinoma. Clin. Transl. Oncol.
14(10): 783-790.

Kumar, S. S., R. Holla, P. Sukumar, S. Padmanabanand N. Vivekanandan. 2013. Treatment planning and dosimetric comparison study on two different volumetric modulated arc therapy delivery techniques. Rep. Pract. Oncol. Radiother. 18(2): 87-94.

Lin, A., H. M. Kim, J. E. Terrell, L. A. Dawson, J. A. Ship and A. Eisbruch. 2003. Quality of life after parotid-sparing IMRT for head-and-neck cancer: A prospective longitudinal study. Int. J. Radiat. Oncol. Biol. Phys. 57(1): 61-70.

Moret, J. A., O. Koelbl and L. Bogner. 2009. Quasi-IMAT technique and secondary cancer risk in prostate cancer. Strahlenther. Onkol. 185(4): 248-253.

Navarria, P., G. Reggiori, F. Pessina, A. M. Ascolese, S. Tomatis, P. Mancosu, F. Lobefalo, E. Clerici, E. Lopci and A. Bizzi. 2014. Investigation on the role of integrated PET/MRI for target volume definition and radiotherapy planning in patients with high grade glioma. Radiother. Oncol. 112(3): 425-429.

Nutting, C. M., J. P. Morden, K. J. Harrington, T. G. Urbano, S. A. Bhide, C. Clark, E. A. Miles, A. B. Miah, K. Newbold and M. Tanay. 2011. Parotid-sparing intensity modulated versus conventional radiotherapy in head and neck cancer (PARSPORT): A phase 3 multicentre randomised controlled trial. Lancet Oncol. 12(2): 127-136.

Scott-Brown, M., A. Miah, K. Harrington and C. Nutting. 2010. Evidencebased review: Quality of life following head and neck intensitymodulated radiotherapy. Radiother. Oncol. 97(2): 249-257.

Spirou, S. V. and C. S. Chui. 1998. A gradient inverse planning algorithm with dose-volume constraints. Med. Phys. 25(3): 321-333.

Sun, X., S. Su, C. Chen, F. Han, C. Zhao, W. Xiao, X. Deng, S. Huang, C. Lin and T. Lu. 2014. Long-term outcomes of intensitymodulated radiotherapy for 868 patients with nasopharyngeal carcinoma: An analysis of survival and treatment toxicities. Radiother. Oncol. 110(3): 398-403.

Tejpal, G., A. Jaiprakash, B. Susovan, S. Ghosh-Laskar, V. Murthy and A. Budrukkar. 2010. IMRT and IGRT in head and neck cancer: Have we delivered what we promised? Indian J. Surg. Oncol. 1(2): 166-185.

Wu, Q., R. Mohan, M. Morris, A. Lauve and R. Schmidt-Ullrich. 2003. Simultaneous integrated boost intensity-modulated radiotherapy for locally advanced head-and-neck squamous cell carcinomas. I: Dosimetric results. Int. J. Radiat. Oncol. Biol. Phys. 56(2): 573-585.

Xie, X., S. Ouyang, H. Wang, W. Yang, H. Jin, B. Hu and L. Shen. 2014 Dosimetric comparison of left-sided whole breast irradiation with 3D-CRT, IP-IMRT and hybrid IMRT. Oncol. Rep. 31: 2195-2205.

Yoon, M., S. Y. Park, D. Shin, S. B. Lee, H. R. Pyo, D. Y. Kim and K. H. Cho. 2007. A new homogeneity index based on statistical analysis of the dose-volume histogram. J. Appl. Clin. Med. Phys. 8(2): 9-17.

Zelefsky, M. J., Z. Fuks, M. Hunt, Y. Yamada, C. Marion, C. C. Ling, H. Amols, E. S. Venkatraman and S. A. Leibel. 2002. High-dose intensity modulated radiation therapy for prostate cancer: Early toxicity and biochemical outcome in 772 patients. Int. J. Radiat. Oncol. Biol. Phys. 53(5): 1111-1116.

Zhou, Q., Y. He, Y. Zhao, Y. Wang, W. Kuang and L. Shen. 2017. A study of 358 cases of locally advanced nasopharyngeal carcinoma receiving intensity-modulated radiation therapy: Improving the seventh edition of the American joint committee on cancer T-staging system. Biomed Res. Int. 2017;2017: 1419676.

Zwahlen, D. R., J. D. Ruben, P. Jones, F. Gagliardi, J. L. Millar and U. Schneider. 2009. Effect of intensity-modulated pelvic radiotherapy on second cancer risk in the postoperative treatment of endometrial and cervical cancer. Int. J. Radiat. Oncol. Biol. Phys. 74(2): 539-545. 